\title{
Passivation of copper in silicon by hydrogen
}

\author{
C. D. Latham* and M. Alatalo \\ Department of Electrical Engineering, Lappeenranta University of Technology, P.O. Box 20, FIN-53851 Lappeenranta, Finland \\ R. M. Nieminen \\ Laboratory of Physics, Helsinki University of Technology, P.O. Box 1100, FIN-02015 HUT, Finland \\ R. Jones \\ School of Physics, University of Exeter, Exeter, EX4 4QL, United Kingdom \\ S. Öberg \\ Department of Mathematics, Luleå University of Technology, SE-97187 Luleå, Sweden \\ P. R. Briddon \\ Physics Centre, School of Natural Science, University of Newcastle upon Tyne, Newcastle, NE1 7RU, United Kingdom
}

(Received 27 May 2005; published 20 December 2005)

\begin{abstract}
The structures and energies of model defects consisting of copper and hydrogen in silicon are calculated using the AIMPRO local-spin-density functional method. For isolated copper atoms, the lowest energy location is at the interstitial site with $T_{d}$ symmetry. Substitutional copper atoms are found to adopt a configuration with $D_{2 d}$ symmetry. We conclude that the symmetry is lowered from $T_{d}$ due to the Jahn-Teller effect. Interstitial hydrogen atoms are found to bind strongly to substitutional copper atoms with an energy that is more than the difference in formation energy over the interstitial site for $\mathrm{Cu}$. The resulting complex has $C_{2 v}$ symmetry in the -2 charge state where the $\mathrm{H}$ atom is situated about $1.54 \AA$ away from the $\mathrm{Cu}$ atom in a [100] direction. In other charge states the symmetry of the defect is lowered to $C_{s}$ or $C_{1}$. A second hydrogen atom can bind to this complex with nearly the same energy as the first. Two structures are found for copper dihydride complexes that have nearly equal energies; one with $C_{2}$ symmetry, and the other with $C_{s}$ symmetry. The binding energy for a third hydrogen atom is slightly more than for the first. Calculated electronic levels for the model defects relative to one another are found to be in fair to good agreement with experimental data, except for the copper-dihydride complex. The copper trihydride complex has no deep levels in the bandgap, according to our calculations.
\end{abstract}

DOI: 10.1103/PhysRevB.72.235205

PACS number(s): 61.72.Bb, 61.72.Ji, 61.72.Tt, 71.15.Mb

\section{INTRODUCTION}

Copper is well known for its remarkable ability to diffuse through silicon very easily as interstitial atoms. The activation energy is measured to be only $0.18 \pm 0.01 \mathrm{eV}$ (Ref. 1). It can also replace silicon atoms in the form of substitutional defects $\mathrm{Cu}_{\mathrm{Si}}$, and form complexes with impurities and silicon vacancies. The electrical levels of various copper related defects are known from experiment. ${ }^{2-7}$ Analysis and interpretation of the observations is assisted by the fact that related impurities and their complexes with hydrogen such as zinc, ${ }^{8}$ silver, ${ }^{9}$ gold, ${ }^{10,11}$ platinum, ${ }^{12}$ and palladium ${ }^{13}$ have defect induced levels of similar character. According to Watkins, the electrical levels of substitutional transition metals are derived from those of the silicon monovacancy $V_{\mathrm{Si}}$ (Ref. 14). In this model, the $d$ states of a transition metal impurity, deep in the valence band, perturb the $t_{2}$ gap levels of ideal, $T_{d}$-symmetry $V_{\mathrm{Si}}$. Hydrogen atoms behave as donors or acceptors, depending on the electron chemical potential $\mu_{e}$ or Fermi level. Thus, the ionization energies of hydrogenated substitutional transition metals in silicon are also derived from those of $V_{\mathrm{Si}}$. The experiments mentioned previously all employ thermalelectrical techniques to measure the energies of the electrical levels, namely, deep level transient spectroscopy (DLTS),
Laplace DLTS, and minority carrier transient spectroscopy (MCTS). However, these methods provide very little direct information about what form these defects take.

The technological interest that motivates our work comes from the increasing use of copper instead of aluminum for electrical connections on silicon-based microelectronic devices. Copper gives superior performance, except for the problem of diffusion where elaborate schemes must be employed to prevent contamination of silicon devices by the metal. One such scheme is to trap copper atoms in stable defects that do not interfere with device operation. It is, therefore, crucial to model the properties of these defects at the atomic scale. In the work reported here we use an accurate method based on the local-spin-density approximation of density functional theory.

In previous theoretical studies using local-density, localspin-density, and Hartree-Fock approximations, Estreicher and Hastings, ${ }^{15}$ Estreicher, ${ }^{16,17}$ Estreicher et al., ${ }^{18}$ and West et al. ${ }^{19}$ considered defects involving various combinations of $\mathrm{Cu}, \mathrm{O}, \mathrm{H}$, and vacancies in $\mathrm{Si}$. Their work focused on the formation energetics, structures, and dynamics of the defects. The main emphasis in this work is on the electrical levels of substitutional copper and copper-hydrogen complexes in $\mathrm{Si}$. 
We will also compare our results for formation energetics and structures with those of Refs. 15-19.

\section{METHOD}

\section{A. Total energy calculations}

Model defects are constructed in supercells with cubic crystal symmetry, where the ideal Si structure contains either 64 or 216 atoms. In other words, the pure Si supercells consist of either $2 \times 2 \times 2$ or $3 \times 3 \times 3$ conventional cubic unit cells stacked together. Their total energies are calculated using a program package based on self-consistent spinpolarized local-density-functional theory, AIMPRO. ${ }^{20-22}$ It can provide detailed information about the structure of defects, their formation energies, electronic band structure, local vibrational modes, etc. The method uses a Gaussian basis set to describe the Kohn-Sham wave functions of the valence electrons, while the charge density is represented by a plane wave basis in reciprocal space, together with the MonkhorstPack (MP) scheme to sample the band structure. ${ }^{23}$ Core electrons are replaced by a pseudopotential constructed using the Hartwigsen-Goedecker-Hutter (HGH) scheme. ${ }^{24}$ The exchange-correlation energy contribution is evaluated according to the formula described by Perdew and Wang. ${ }^{25}$

Selected tests are then performed to ensure that the various choices and approximations made for the particular problem under consideration are valid. To achieve satisfactory results it is necessary to have four Gaussian functions per atom for the wave-function basis. Orbitals with $s, p$, and $d$ symmetry are generated by appropriate prefactors. For $\mathrm{Si}$ atoms, $d$ orbitals are only needed for the two smallest Gaussians, and $\mathrm{H}$ atoms require none. The plane wave basis kinetic energy cutoff $E_{\text {cut }}$ that is required to yield converged total energies depends on the types of atoms present. For $\mathrm{Si}$ atoms only, this is $E_{\text {cut }} \sim 80-120 \mathrm{Ry}$. When $\mathrm{H}$ atoms are included, it is necessary to increase this to $E_{\text {cut }} \sim 300-450$ Ry. $\mathrm{Cu}$ atoms need the cutoff energy set to $E_{\mathrm{cut}} \sim 450-675 \mathrm{Ry}$, due to the rapidly varying wave functions of their $3 d$ electrons. The MP sampling mesh required depends on the size of the supercell. For the smaller, $2 \times 2 \times 2$ supercells we use MP- $4^{3} \mathbf{k}$ points, while for the larger $3 \times 3 \times 3$ supercells we use MP- $2^{3} \mathbf{k}$ points. The mesh is shifted to avoid the $\Gamma$ point and folded according to the symmetry of the system. These values for $E_{\text {cut }}$ and MP $\mathbf{k}$ points yield total energies that in purely numerical terms are converged to $\lesssim 10^{-5} \mathrm{Ry}$ or $\lesssim 10^{-4} \mathrm{eV}$.

Defect formation energies are calculated by the conventional method as described by previous authors. ${ }^{26-30}$ In general terms, for defect in charge state $q$ its formation energy is defined as

$$
E_{f}(q)=E_{d}(q)+q \mu_{e}-\sum_{i} n_{i} \mu_{i}
$$

where $E_{d}(q)$ is the total energy of a supercell containing a defect composed from $n_{i}$ atoms of type $i$ with chemical potential $\mu_{i}$, and where $\mu_{e}$ is the electron chemical potential with respect to the top of the valence band of the pure material. The occupancies of defect-induced electrical levels in the bandgap are set by including the appropriate number of electrons in the calculation and keeping the net charge neutral to prevent the total energy from being divergent by applying a uniform background charge of equal and opposite magnitude to the sum of electrons and protons.

\section{B. Electrical levels}

The supercell formalism performs poorly at calculating absolute values for the energies of defect levels. Various remedies have been suggested, but none have yet proved to be entirely satisfactory.

The first problem before Eq. (1) can be applied is to determine the potential of the system, which has no straightforward reference. One method is to estimate the average potential of the supercell sufficiently far from any defect it may contain. Unfortunately, it is often not clear whether a suitable region exists in a supercell where such an average may be taken. The most common method to fix the potential involves aligning the electronic bands of the supercells, plotted as graphs. This procedure normally works alright, but fails when the chosen bands (usually the top of the valence band; often the bottom of the valence band) are confused with defect-induced bands. Even for experienced hands, this pitfall is often not obvious, and when detected it is usually difficult to find an alternative route.

A further, small correction may be necessary to allow for the fact that an electronic transition probably occurs at a point in the Brillouin zone (typically $\Gamma$ ) that does not coincide with any point in the sampling mesh.

Then there is the problem that finite charge distributions in a supercell interact with their images. The resulting energy contribution can be estimated by adding a Madelung correction to Eq. (1) (Ref. 31). This is a sum of terms of which the monopole energy is $E_{\mathrm{M}}(q)=\alpha_{\mathrm{M}} q^{2} / L \epsilon$; where $\alpha_{\mathrm{M}}$ is the Madelung constant, $L$ is the lattice parameter, and $\epsilon$ is the permittivity of the material. The main problems with this correction are that convergence is slow with respect to the cell size, and being proportional to $q^{2}$ makes it unreliable for $|q|>1$. The permittivity is also difficult to calculate accurately $a b$ initio, and normally one must resort to using measured values. The Madelung constant, at least, depends only on the geometry of the supercell.

To avoid these problems, the method that we adopt, therefore, is one that depends only on total energies without any further corrections applied. It provides results in a practical and consistent form in the following way. First, we define for a defect state labeled $d$ with charge states $q$ and $q^{\prime}$ a configuration energy per electron $C_{d}\left(q / q^{\prime}\right)$ that is simply the difference in the total energy divided by the difference in charge between the two states, so that

$$
C_{d}\left(q / q^{\prime}\right)=\left[E_{d}\left(q^{\prime}\right)-E_{d}(q)\right] /\left(q-q^{\prime}\right) .
$$

Note that the structure of the defect in charge state $q$ is not necessarily the same as when it is in charge state $q^{\prime}$.

The configuration energies of $d$ can then be compared with the configuration energies of a chosen reference state or "marker" labeled $m$. This may be either another defect, or a supercell without any defect in it. The energy per electron of 
the state $d$ relative to the reference state $m$ is then

$$
D_{d}\left(q / q^{\prime}\right)=C_{d}\left(q / q^{\prime}\right)-C_{m}\left(p / p^{\prime}\right) .
$$

If $m$ is a defect that has a measured electronic transition energy per electron $E_{m}\left(p / p^{\prime}\right)$ between charge state $p$ and $p^{\prime}$, then it is possible to estimate the energy of a defect level $E_{d}\left(q / q^{\prime}\right)$

$$
E_{d}\left(q / q^{\prime}\right) \approx D_{d}\left(q / q^{\prime}\right)+E_{m}\left(p / p^{\prime}\right) .
$$

Otherwise, when $m$ is a supercell without any defect in it, $C_{m}(0 /+1)=E_{v}$, the energy level of the top of the valence band, while $C_{m}(-1 / 0)=E_{c}$, the energy level of the bottom of the conduction band. Note that the bands of the charged, pure $\mathrm{Si}$ supercells are occupied according to a metallic filling scheme. ${ }^{32}$ It is also necessary to increase the density of the band-structure sampling to MP- $6^{3}$ for 64 -atom supercells and $\mathrm{MP}-4^{3}$ for 216 -atom supercells.

This method for calculating the energies of electrical levels relies on the cancellation of systematic errors and, therefore, is more likely to give best results when $d$ and $m$ are most similar in their electronic character, symmetry, structure, and so on. For example, if both are substitutional transition metals that are near to one another in the periodic table such as $\mathrm{Ag}$ and $\mathrm{Au}$, then estimates of the levels of one with respect to the other are likely to be more accurate than if the reference state is a dissimilar defect. Moreover, while there is no particular restriction on what $p, q, p^{\prime}$, and $q^{\prime}$ are, the best results are expected when both $p=q$ and $p^{\prime}=q^{\prime}$. Naturally, comparing the levels of one defect with another depends on there being a suitable defect to make the comparison with, and it means that the calculation is no longer truly $a b$ initio. If an $a b$ initio result is wanted, then $m$ must be a pure $\mathrm{Si}$ supercell without any defect.

Based on past experience, differences in configuration energies between different charge states of similar defects $D_{d}\left(q / q^{\prime}\right)$ typically have errors $\sim \pm 0.1 \mathrm{eV}$. In favorable circumstances this error can be smaller. The worst case is expected to be when the reference state $m$ is a pure, ideal $\mathrm{Si}$ supercell: then the error may be larger, but perhaps no worse than by a factor of two. Absolute formation energies tend to be of similar accuracy, while differences in energies between similar structures in the same charge state are normally an order of magnitude more accurate. It is difficult to be more precise than this without being unduly pessimistic or overly optimistic. Opinions vary widely on this subject. To guard against rounding errors we quote configuration energies with more digits than the real accuracy of the underlying theoretical approximations. This facilitates possible future reuse or reanalysis of the results.

\section{RESULTS AND DISCUSSION}

\section{A. General observations}

In small supercells the concentration of defects considered here is very much larger than in reality where it is close to the infinitely dilute limit; therefore, to simulate the true situation we choose to fix the lattice parameter $a$ describing supercells to the value that minimizes the total energy of
TABLE I. Configuration energies $C_{d}\left(q / q^{\prime}\right)$ in electron volts for silicon supercells without any defect. The quoted precision, while being numerically significant, is probably at least an order of magnitude better than the theoretical approximations provide. This is to guard against propagation of rounding errors in any subsequent reuse.

\begin{tabular}{lll}
\hline \hline$d \backslash q / q^{\prime}$ & $0 /+1$ & $-1 / 0$ \\
\hline Si 64-atom supercell & 5.037 & 6.023 \\
Si 216-atom supercell & 5.175 & 5.884 \\
\hline \hline
\end{tabular}

pure $\mathrm{Si}$. For the record, this is calculated to be $a=5.3948 \AA$ or $99.33 \%$ of the observed value. The calculated bulk modulus is $B_{0}=97.0 \mathrm{GPa}$ (observed $B_{0}=97.7 \mathrm{GPa}$ ).

Several types of defects are considered in the work reported here: copper-hydrogen complexes; isolated substitutional copper atoms; isolated interstitial copper, and isolated interstitial hydrogen atoms. For the defects involving hydrogen, and for substitutional copper, several different structures are compared.

When $d$ and $m$ are identical supercells of pure silicon without any defects, then $C_{d}(-1 / 0)-C_{m}(0 /+1)$ is the theoretical bandgap $E_{g}$ of the system. This depends on the cell size. The calculated configuration energies for the two sizes of supercell used in this work are given in Table I. For the smaller supercell $E_{g}=6.023-5.037 \approx 0.99 \mathrm{eV}$, while for the larger supercell $E_{g}=5.884-5.175 \approx 0.71 \mathrm{eV}$. This effect is a consequence of the fact that the carrier concentration in a charged supercell of volume $V$ varies as $1 / V$ and the dispersion decreases as cell size increases. Hence, $E_{g}$ converges on the local-density approximation (LDA) value (which in the case of $\mathrm{Si}$ is about half the true value) as the supercell size grows.

If pure silicon supercells are used as the reference configuration energies $C_{m}\left(p / p^{\prime}\right)$ for calculating the ionization energies of defects, then it is evident that this leads to another problem when the real ionization energy is more than the theoretical bandgap of the supercell but still less than the real bandgap of the crystal. In cases where this occurs we need to adopt a different strategy. If it is known or judged that a level falls into this category, then, ionization energies for donors are calculated with respect to $E_{c}$ [i.e., $C_{m}(-1 / 0)$, where $m=\mathrm{Si}$ instead of $E_{v}$, and the other way around for acceptors. Making this judgement for levels about which little or nothing is known is going to be a subjective decision, but in the absence of a better theory, there is no alternative.

\section{B. Isolated hydrogen}

Isolated interstitial hydrogen in silicon $\mathrm{H}_{i}$ has been studied in great detail in the past by many experimental and theoretical methods: see, for example, Refs. 33-36. See also the recent study of muonium in Si by Lord et al. ${ }^{37}$ Thus, in our calculations isolated hydrogen represents a known reference state. $\mathrm{H}_{i}$ induces an electronic level in the bandgap that may be occupied with zero, one, or two electrons depending on where the position of the Fermi level $\mu_{e}$ is with respect to 
the defect state. As a bare proton $\mathrm{H}_{i}^{+1}$, or a neutral atom $\mathrm{H}_{i}^{0}$, its lowest energy location is at the bond-centered site between silicon atoms, while in the -1 charge state it prefers to be situated at or near the tetrahedral interstitial site, away from regions of high electron density. In the bond-centered state, the $\mathrm{Si}-\mathrm{Si}$ distance is about $40 \%$ larger for a bond containing a proton than the bulk value.

The calculated formation energy of the neutral bondcentered defect $\left(\mathrm{H}_{i}\right)_{\mathrm{bc}}^{0}$ in the smaller and larger size supercells, respectively, is 1.47 and $1.42 \mathrm{eV}$ relative to to isolated hydrogen molecules and bulk silicon. A neutral hydrogen atom at the tetrahedral interstitial site $\left(\mathrm{H}_{i}\right)_{T_{d}}^{0}$ is estimated to be higher in energy by $0.12 \mathrm{eV}$ by our method. This energy difference is $1.08 \mathrm{eV}$ in the +1 charge state, while in the -1 state the situation is reversed and the $\left(\mathrm{H}_{i}\right)_{T_{d}}^{-1}$ defect is lower in energy by $0.66 \mathrm{eV}$ relative to $\left(\mathrm{H}_{i}\right)_{\mathrm{bc}}^{-1}$.

\section{Electrical levels of isolated hydrogen}

The gross change in structure with charge state for $\mathrm{H}_{i}$ gives it the special property that the donor level lies above the acceptor level; hence, the quantity $U=E_{d}(-1 / 0)-E_{d}(0 /$ $+1)$ is negative and the neutral state is unstable. Consequently, it is difficult to measure the electrical levels for $\mathrm{H}_{i}$; its concentration in the neutral state is very low. Two fairly recent measurements of the donor level $E_{d}(0 /+1)$ of interstitial hydrogen $\left(d=\mathrm{H}_{i}\right)$ in silicon place it at $E_{c}-0.175 \mathrm{eV}$ and $E_{c}-0.16 \mathrm{eV}$ (Refs. 35 and 36). The change in structure that occurs for the reaction $\mathrm{H}_{i}^{-1} \rightarrow \mathrm{H}_{i}^{0}+e$ further complicates measurement of the acceptor level. It is estimated to be about $0.46 \mathrm{eV}$ below the donor level placing it near midgap at $E_{c}-0.62 \mathrm{eV}$ (Ref. 36).

Three sets of calculated configuration energies for $d=\mathrm{H}_{i}$ are given in Table II. In the first two cases the defect is constrained to be in a tetrahedral interstitial structure $\left(\mathrm{H}_{i}\right)_{T_{d}}$ or the bond-centred form $\left(\mathrm{H}_{i}\right)_{\mathrm{bc}}$ only. The third row of figures shows $C_{d}\left(q / q^{\prime}\right)$ calculated for $\mathrm{H}_{i}$ in its lowest energy states, $\left(\mathrm{H}_{i}\right)_{T_{d}}^{-1},\left(\mathrm{H}_{i}\right)_{\mathrm{bc}}^{0}$, and $\left(\mathrm{H}_{i}\right)_{\mathrm{bc}}^{+1}$.

Using $C_{m}(-1 / 0)$ where $m=\mathrm{Si}$ from Table $\mathrm{I}$ as our reference makes the calculated donor level $E_{d}(0 /+1)$ $=6.023-5.913 \approx E_{c}-0.11 \mathrm{eV}$ for the smaller supercell, and $E_{d}(0 /+1)=5.884-5.817 \approx E_{c}-0.07 \mathrm{eV}$ for the larger supercell. Note that because this is a donor with an energy with respect to $E_{v}$ well above the theoretical bandgap, we need to use $E_{c}$ as the reference. Our calculated acceptor level, which includes the change in structure, is $E_{d}(-1 / 0) \approx E_{c}-0.71 \mathrm{eV}$ for the smaller supercell and $E_{d}(-1 / 0) \approx E_{c}-0.50 \mathrm{eV}$ for the larger supercell. This means that $U \approx-0.60 \mathrm{eV}$ in the smaller supercell, and $U \approx-0.44 \mathrm{eV}$ in the larger supercell.

If we assume that the transition state is neutral hydrogen at a tetrahedral interstitial site $\left(\mathrm{H}_{i}\right)_{T}^{0}$, then the activation energy for the reaction via this state is $0.83 \mathrm{eV}$ for the smaller supercell and $0.86 \mathrm{eV}$ for the larger supercell. Problems with finding a self-consistent total energy for $\left(\mathrm{H}_{i}\right)_{T_{d}}^{0}$ in the larger supercell mean there is some additional uncertainty in the latter figure. The measured activation energy is $0.84 \mathrm{eV}$ (Ref. 36).
TABLE II. Configuration energies $C_{d}\left(q / q^{\prime}\right)$ in electron volts for isolated interstitial hydrogen in silicon. The same remarks about numerical versus real precision as in Table I and Sec. II apply here.

\begin{tabular}{lcrcc}
\hline \hline & \multicolumn{2}{c}{$2 \times 2 \times 2$ supercell } & \multicolumn{2}{c}{$3 \times 3 \times 3$} \\
& $0 /+1$ & $-1 / 0$ & $0 /+1$ & $-1 / 0$ \\
\hline$\left(\mathrm{H}_{i}\right)_{T_{d}}$ & 4.953 & 5.192 & - & $\approx 5.03$ \\
$\left(\mathrm{H}_{i}\right)_{\mathrm{bc}}$ & 5.913 & 5.974 & 5.817 & 5.885 \\
$\mathrm{H}_{i}$ & 5.913 & 5.314 & 5.817 & 5.382 \\
\hline \hline
\end{tabular}

\section{Isolated copper}

A single copper atom can in principle either occupy an interstitial site $\mathrm{Cu}_{i}$ or replace a silicon atom within a Si crystal as $\mathrm{Cu}_{\mathrm{Si}}$. The difference in formation energy between substitutional copper with exactly tetrahedral symmetry $\left(\mathrm{Cu}_{\mathrm{Si}}\right)_{T_{d}}$ and interstital copper at a tetrahedral site $\left(\mathrm{Cu}_{i}\right)_{T_{d}}$ depends on the charge state of the defects. When both are neutral, interstitial $\left(\mathrm{Cu}_{i}\right)_{T_{d}}^{0}$ is calculated to be lower in energy than substitutional $\left(\mathrm{Cu}_{\mathrm{Si}}\right)_{T_{d}}^{0}$ by $0.70 \mathrm{eV}$. In the +1 charge state the amount increases to $1.44 \mathrm{eV}$, while in the -1 state it is only $0.04 \mathrm{eV}$. Although the intersitial has a lower formation energy, both forms are observed to exist. The net reaction that changes $\mathrm{Cu}_{\mathrm{Si}}$ into $\mathrm{Cu}_{i}$ creates a silicon vacancy $V_{\mathrm{Si}}$ which costs considerably more energy that the amount gained. The absolute formation energy $E_{f}$ of neutral substitutional copper $\left(\mathrm{Cu}_{\mathrm{Si}}\right)_{T_{d}}^{0}$ with respect to pure silicon and copper, is calculated to be $2.30 \mathrm{eV}$ in the smaller size supercell containing 64 atoms, and $2.40 \mathrm{eV}$ in the larger size supercell containing 216 atoms.

Substitutional copper with $T_{d}$ symmetry, in the neutral charge state, has a doubly degenerate $t_{2}$ level occupied by three electrons. This raises the possibility that it may undergo a symmetry lowering Jahn-Teller distortion that splits the orbital degeneracy into two levels of $a_{1}$ and $e$ character. The effect is not expected to be large, and any energy minima fairly shallow. To examine the problem, several different initial structures with different symmetries are used in addition to the ones with $T_{d}$ symmetry. The AIMPRO computer code can either preserve the symmetry of the initial structure when minimizing the total energy, or perform an unconstrained optimization as required. The relaxation pattern when using unconstrained optimization in 64 atom supercells appears to result in a structure with approximately $D_{2 d}$ symmetry in neutral and negative charge states.

There are two ways that a distortion with $D_{2 d}$ symmetry may occur. The pattern found here is such that for the six $\mathrm{Si}-\mathrm{Si}$ lengths between the four $\mathrm{Si}$ atoms neighboring the $\mathrm{Cu}$ atom, there are four equivalent lengths which are shorter than the other two, that in turn are equivalent to each other. In other words, the bounding box for this structure with its six faces in the cubic $\{100\}$ planes is short and broad in shape rather than long and thin. For convenience we will label this $D_{2 d}(A)$. The other form, which we will consider later, has the four equivalent $\mathrm{Si}-\mathrm{Si}$ lengths longer than the other pair of equal lengths. Its bounding box in the six cubic $\{100\}$ planes is long and thin; we will label this form $D_{2 d}(B)$. 
TABLE III. Calculated $\mathrm{Cu}-\mathrm{Si}$ and $\mathrm{Si}-\mathrm{Si}$ lengths (ångströms) and relative energies $\Delta E$ (electron volts) for $\mathrm{Cu}_{\mathrm{Si}}^{q}$ with respect to $\left(\mathrm{Cu}_{\mathrm{Si}}\right)_{T_{d}}^{q}$ in $2 \times 2 \times 2,64$-atom and $3 \times 3 \times 3,216$-atom supercells. The six Si-Si lengths are the six edges of the tetrahedron containing the Cu atom. The calculated $\mathrm{Si}-\mathrm{Si}$ bond length of pure $\mathrm{Si}$ is $2.336 \AA$.

\begin{tabular}{|c|c|c|c|c|c|c|c|c|c|c|c|}
\hline$q$ & $\mathrm{Cu}-\mathrm{Si}$ & $\mathrm{Cu}-\mathrm{Si}$ & $\mathrm{Cu}-\mathrm{Si}$ & $\mathrm{Cu}-\mathrm{Si}$ & $\mathrm{Si}-\mathrm{Si}$ & $\mathrm{Si}-\mathrm{Si}$ & $\mathrm{Si}-\mathrm{Si}$ & $\mathrm{Si}-\mathrm{Si}$ & $\mathrm{Si}-\mathrm{Si}$ & $\mathrm{Si}-\mathrm{Si}$ & $\Delta E$ \\
\hline \multicolumn{12}{|c|}{64 atoms, $T_{d}$ configuration } \\
\hline+1 & 2.298 & 2.298 & 2.298 & 2.298 & 3.752 & 3.752 & 3.752 & 3.752 & 3.752 & 3.752 & 0.000 \\
\hline 0 & 2.271 & 2.271 & 2.271 & 2.271 & 3.708 & 3.708 & 3.708 & 3.708 & 3.708 & 3.708 & 0.000 \\
\hline-1 & 2.247 & 2.247 & 2.247 & 2.247 & 3.669 & 3.669 & 3.669 & 3.669 & 3.669 & 3.669 & 0.000 \\
\hline-2 & 2.228 & 2.228 & 2.228 & 2.228 & 3.638 & 3.638 & 3.638 & 3.638 & 3.638 & 3.638 & 0.000 \\
\hline \multicolumn{12}{|c|}{64 atoms, unconstrained configuration } \\
\hline+1 & 2.298 & 2.298 & 2.298 & 2.298 & 3.749 & 3.752 & 3.752 & 3.753 & 3.755 & 3.757 & -0.000 \\
\hline 0 & 2.273 & 2.273 & 2.273 & 2.273 & 3.682 & 3.683 & 3.686 & 3.688 & 3.764 & 3.767 & -0.007 \\
\hline-1 & 2.248 & 2.249 & 2.250 & 2.250 & 3.614 & 3.615 & 3.619 & 3.621 & 3.781 & 3.782 & -0.047 \\
\hline-2 & 2.229 & 2.229 & 2.230 & 2.230 & 3.614 & 3.617 & 3.617 & 3.619 & 3.688 & 3.688 & -0.012 \\
\hline \multicolumn{12}{|c|}{216 atoms, $T_{d}$ configuration } \\
\hline+1 & 2.278 & 2.278 & 2.278 & 2.278 & 3.719 & 3.719 & 3.719 & 3.719 & 3.719 & 3.719 & 0.000 \\
\hline 0 & 2.266 & 2.266 & 2.266 & 2.266 & 3.793 & 3.793 & 3.793 & 3.793 & 3.793 & 3.793 & 0.000 \\
\hline-1 & 2.251 & 2.251 & 2.251 & 2.251 & 3.676 & 3.676 & 3.676 & 3.676 & 3.676 & 3.676 & 0.000 \\
\hline-2 & 2.232 & 2.232 & 2.232 & 2.232 & 3.644 & 3.644 & 3.644 & 3.644 & 3.644 & 3.644 & 0.000 \\
\hline \multicolumn{12}{|c|}{216 atoms, $D_{2 d}(A)$ configuration } \\
\hline+1 & 2.277 & 2.277 & 2.277 & 2.277 & 3.693 & 3.693 & 3.693 & 3.693 & 3.766 & 3.766 & +0.000 \\
\hline 0 & 2.266 & 2.266 & 2.266 & 2.266 & 3.641 & 3.641 & 3.641 & 3.641 & 3.815 & 3.815 & -0.049 \\
\hline-1 & 2.251 & 2.251 & 2.251 & 2.251 & 3.589 & 3.589 & 3.589 & 3.589 & 3.842 & 3.842 & -0.151 \\
\hline-2 & 2.310 & 2.310 & 2.310 & 2.310 & 3.600 & 3.600 & 3.600 & 3.600 & 3.733 & 3.733 & -0.042 \\
\hline \multicolumn{12}{|c|}{216 atoms, $D_{2 d}(B)$ configuration } \\
\hline+1 & 2.279 & 2.279 & 2.279 & 2.279 & 3.616 & 3.616 & 3.774 & 3.774 & 3.774 & 3.774 & -0.008 \\
\hline 0 & 2.268 & 2.268 & 2.268 & 2.268 & 3.595 & 3.595 & 3.756 & 3.756 & 3.756 & 3.756 & -0.036 \\
\hline-1 & 2.251 & 2.251 & 2.251 & 2.251 & 3.581 & 3.581 & 3.723 & 3.723 & 3.723 & 3.723 & -0.060 \\
\hline-2 & 2.233 & 2.233 & 2.233 & 2.233 & 3.591 & 3.591 & 3.673 & 3.673 & 3.673 & 3.673 & -0.020 \\
\hline \multicolumn{12}{|c|}{216 atoms, $C_{2 v}$ configuration } \\
\hline+1 & 2.279 & 2.279 & 2.279 & 2.279 & 3.608 & 3.614 & 3.776 & 3.776 & 3.776 & 3.776 & -0.008 \\
\hline 0 & 2.256 & 2.256 & 2.278 & 2.278 & 3.530 & 3.704 & 3.741 & 3.741 & 3.741 & 3.741 & -0.023 \\
\hline-1 & 2.226 & 2.226 & 2.270 & 2.270 & 3.391 & 3.697 & 3.697 & 3.697 & 3.697 & 3.778 & -0.059 \\
\hline-2 & 2.221 & 2.221 & 2.243 & 2.243 & 3.532 & 3.656 & 3.656 & 3.656 & 3.656 & 3.703 & -0.023 \\
\hline \multicolumn{12}{|c|}{216 atoms, unconstrained configuration } \\
\hline+1 & 2.278 & 2.278 & 2.278 & 2.278 & 3.607 & 3.607 & 3.773 & 3.773 & 3.775 & 3.780 & -0.008 \\
\hline 0 & 2.264 & 2.265 & 2.267 & 2.267 & 3.632 & 3.638 & 3.643 & 3.651 & 3.808 & 3.824 & -0.049 \\
\hline-1 & 2.249 & 2.250 & 2.250 & 2.250 & 3.583 & 3.585 & 3.593 & 3.595 & 3.834 & 3.841 & -0.151 \\
\hline-2 & 2.233 & 2.233 & 2.233 & 2.233 & 3.598 & 3.598 & 3.603 & 3.604 & 3.725 & 3.743 & -0.042 \\
\hline
\end{tabular}

The energy difference between the unconstrained structure and the one with exact $T_{d}$ symmetry is greatest in the -1 charge state: the amount is nearly $0.05 \mathrm{eV}$. In the neutral state the energy difference is about one twelfth of the size, while in the -2 charge state it is about six times smaller than in the -1 charge state. No significant distortion occurs in the +1 charge state: the unconstrained optimization and the $T_{d}$ symmetry system both have essentially the same total energy and structure. In all charge states the $\mathrm{Cu}-\mathrm{Si}$ distance is smaller than the bulk $\mathrm{Si}-\mathrm{Si}$ bond length. The more electrons the system has, the greater the difference becomes.

It is well established both in theory and by experiment that the related system of $V_{\mathrm{Si}}$ undergoes a similar Jahn-Teller distortion, and that the nature of the distortion given by theory depends on the model used, particularly its size. For example, see Ref. 38. The supercell based on 64 atoms, while being a useful model, is not really sufficiently large to obtain a fully converged result with respect to size. The 216- 
TABLE IV. Configuration energies $C_{d}\left(q / q^{\prime}\right)$ in electron volts for isolated copper in silicon. The same remarks about numerical versus real precision as in Table I and Sec. II apply here.

\begin{tabular}{|c|c|c|c|c|c|c|}
\hline \multirow[b]{2}{*}{$d \backslash q / q^{\prime}$} & \multicolumn{3}{|c|}{$2 \times 2 \times 2$ supercell } & \multicolumn{3}{|c|}{$3 \times 3 \times 3$ supercell } \\
\hline & $0 /+1$ & $-1 / 0$ & $-2 /-1$ & $0 /+1$ & $-1 / 0$ & $-2 /-1$ \\
\hline$\left(\mathrm{Cu}_{i}\right)_{T_{d}}$ & 5.991 & 6.014 & & & & \\
\hline$\left(\mathrm{Cu}_{\mathrm{Si}}\right)_{T_{d}}$ & 5.268 & 5.368 & 5.472 & 5.389 & 5.482 & 5.535 \\
\hline$\left(\mathrm{Cu}_{\mathrm{Si}}\right)_{C_{1}}$ & 5.262 & 5.328 & 5.507 & 5.348 & 5.381 & 5.644 \\
\hline
\end{tabular}

atom supercell, on the other hand, is expected to be large enough. With this in mind, and taking into consideration the results for the 64-atom supercell, we now turn our attention to a more detailed examination of possible distortions for the 216-atom supercell.

When a constrained minimization is performed in a 216atom supercell, both $A$ and $B$ forms with $D_{2 d}$ symmetry are found to be local energy minima in all charge states. Both are lower in energy than $\mathrm{Cu}_{\mathrm{Si}}$ with exact $T_{d}$ symmetry except for the +1 charge state of the $D_{2 d}(A)$ defect which has the same energy as the $T_{d}$ structure. The $D_{2 d}(A)$ form is also lower in energy than the $D_{2 d}(B)$ form except in the +1 charge state.

If the symmetry of the system is lowered to $C_{2 v}$, then the pair of Si-Si lengths that are equal in the $D_{2 d}$ case are now unequal. The two unequal and four equivalent lengths can be ordered in three ways. Our initial, unoptimized structure has one of the unequal lengths shorter than the set of four equivalent ones, and the other is longer. Following optimization, this pattern is retained when the defect has a negative charge, while in the neutral and positive states both of the unequal lengths are shorter than the set of equivalent four. The total energy in all charge states is nearly the same as for the $D_{2 d}(B)$ structure.

Attempts to optimize structures with $C_{3 v}$ symmetry do not yield good results. The algorithms for self-consistency fail, and even with various adjustments to work around the problem, the total energy is always higher than for the exact $T_{d}$ case.

When no symmetry constraints are applied then the optimized structure is essentially the $D_{2 d}(A)$ configuration in the negative and neutral charge states, and matches very closely the $D_{2 d}(B)$ form in the +1 charge state. Their total energies in the unconstrained and constrained calculations are equal to within the numerical accuracy of the method. The formation energy in the lowest energy configuration for neutral substitutional copper in silicon calculated by this method in a 216atom supercell is $E_{f}\left(\mathrm{Cu}_{\mathrm{Si}}^{0}\right) \approx 2.35 \mathrm{eV}$ with respect to fcc copper metal and cubic silicon. Details of these results are given in Table III.

At this point it is necessary to consider some less obvious consequences of the approximations made in these calculations. All essential tests, adjustments, and optimizations have been made with due care and attention. We find that a symmetry lowering structural distortion occurs, apparently due to the Jahn-Teller effect. However, the way that we have sampled the band structure and ignored spin-orbit interactions means that the character and magnitude of the distortion may still be wrong. By following the normal practice of using a sampling mesh that deliberately avoids the gamma point (which would give a poor estimate of the total energy if included) we have already broken the orbital symmetry. This leads to an artificial lowering of the total energy. Having said this, the situation is not as alarming as it might seem. When a supercell is large, the Brillouin zone is small, hence any point in it is near $\Gamma$ where the orbital symmetry is correct. Thus, for a sufficiently large supercell the artificial splitting of the degenerate level and dispersion effects due to the supercell formalism should be negligible. Spin-orbit coupling effects can also lift the orbital degeneracy. As these are not included in our model, this introduces an additional uncertainty, although we expect this to affect things to a lesser degree than the Brillouin zone sampling does. Under most circumstances the factor limiting the accuracy of the method is the local-spin-density approximation itself.

\section{E. Electrical levels of isolated copper}

Isolated substitutional copper in silicon has three electrical levels in the bandgap. Measurements reported over a number of years have provided progressively better estimates of their energies., ${ }^{2,35-7}$ The three levels, according to Knack et al. ${ }^{5-7}$ are a donor at $E_{d}(0 /+1)=E_{v}+0.207 \mathrm{eV}$, an acceptor at $E_{d}(-1 / 0)=E_{v}+0.478 \mathrm{eV}$, and a double acceptor at $E_{d}(-2 /-1)=E_{c}-0.167 \mathrm{eV}$. Isolated interstitial copper has a donor level at $E_{d}(0 /+1)=E_{c}-0.15 \mathrm{eV}$ (Ref. 4).

The calculated configuration energies for $d=\mathrm{Cu}_{i}$ and $d$ $=\mathrm{Cu}_{\mathrm{Si}}$ are given in Table IV. For interstitial copper we have only performed calculations using the smaller size of supercell. The calculated energy of the donor level is $E_{d}(0 /+1)$ $\approx E_{c}-0.03 \mathrm{eV}$. In other words, adding an electron to this system costs very nearly the same energy as adding an electron to a 64-atom supercell of pure silicon. For substitutional copper, symmetry has a larger effect in the larger supercell, and increases as electrons are added to the system. When the symmetry is unconstrained and using $m=\mathrm{Si}$, we arrive at $E_{d}(0 /+1) \approx E_{v}+0.22 \mathrm{eV}, E_{d}(-1 / 0) \approx E_{c}-0.70 \mathrm{eV}$, and $E_{d}(-2 /-1) \approx E_{c}-0.52 \mathrm{eV}$ for the energies of the three electrical levels of $\mathrm{Cu}_{\mathrm{Si}}$ in a 64-atom supercell. Increasing the supercell size to 216 atoms changes these energies to $E_{d}(0 /+1) \approx E_{v}+0.17 \mathrm{eV}, \quad E_{d}(-1 / 0) \approx E_{c}-0.50 \mathrm{eV}, \quad$ and $E_{d}(-2 /-1) \approx E_{c}-0.24 \mathrm{eV}$. Note that in accord with our strategy for estimating electrical levels, the calculated acceptor level $E_{d}(-1 / 0)$ in both cases when expressed with respect to $E_{c}$, is above the top of the valence band. Hence, to facilitate comparison with experiment, we may simply subtract the observed energy for the acceptor level from the measured 

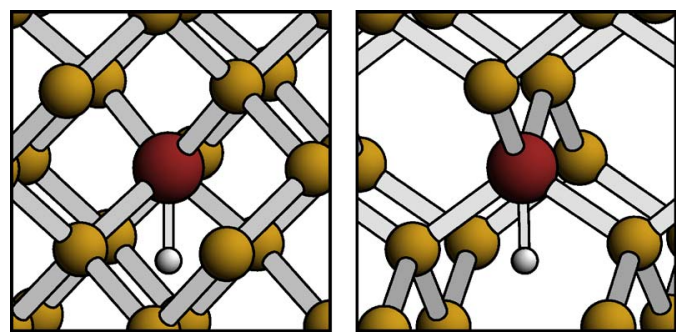

FIG. 1. (Color online) Two views of the $C_{2 v}$ symmetry copper monohydride complex in Si. The unique, large atom near the center is $\mathrm{Cu}$; the small atom directly below it is $\mathrm{H}$; all the others are Si.

bandgap energy $E_{g}=1.17 \mathrm{eV}$. This yields $E_{d}(-1 / 0) \approx E_{c}$ $-0.69 \mathrm{eV}$ for the observed level.

\section{F. Copper-hydrogen complexes}

Copper and hydrogen in silicon can combine to form copper-hydrogen complexes. These have several possible structures and charge states to consider, making this a more complicated problem than the isolated atoms. For example, there are three arrangements with trigonal symmetry that have collinear $\mathrm{Cu}_{\mathrm{Si}}, \mathrm{Si}_{\mathrm{Si}}$, and $\mathrm{H}$ atoms: $\left(\mathrm{Si}_{\mathrm{Si}^{-}} \mathrm{Cu}_{\mathrm{Si}^{-}} \mathrm{H}\right)_{C_{3}}$, $\left(\mathrm{Cu}_{\mathrm{Si}^{-}}-\mathrm{H}_{\mathrm{bc}}-\mathrm{Si}_{\mathrm{Si}}\right)_{C_{3 v}}$, and $\left(\mathrm{Cu}_{\mathrm{Si}^{-}} \mathrm{Si}_{\mathrm{Si}^{-}}-\mathrm{H}\right)_{C_{3 v}}$. It is also possible that a hydrogen atom may be located in some other direction relative to a given $\mathrm{Cu}-\mathrm{Si}$ bond. According to our calculations, the lowest energy structure in all charge states has the $\mathrm{H}$ atom close to a cubic site and directly bonded to the copper. This is located in a [100] direction on a (110) plane between two equivalent $\mathrm{Si}$ atoms. The exact location of the $\mathrm{H}$ atom depends on the charge state of the complex. When the defect is negatively charged then the $\mathrm{H}$ atom is equidistant from the two nearest $\mathrm{Si}$ atoms, and occupies in the same (110) plane as them and the $\mathrm{Cu}$ atom. The defect has $C_{2 v}$ symmetry (see Fig. 1). In the neutral and +1 charge state the minimum energy structure has either $C_{s}$ or $C_{1}$ structure (see Figs. 2 and 3). The energies of these structures are degenerate, and are only very slightly lower than the $C_{2 v}$-symmetry structure. In the neutral state the energy difference is estimated to be only $0.01 \mathrm{eV}$ and in the +1 state the difference is $0.03 \mathrm{eV}$. It is debatable whether or not these amounts are significant.

The energies of the collinear structures with $C_{3 v}$ symmetry are higher in all charge states than the ones with the $\mathrm{H}$ atom close to a cubic site. In the neutral state the energy differences are $0.22 \mathrm{eV}$ for $\left(\mathrm{Si}_{\mathrm{Si}^{-}} \mathrm{Cu}_{\mathrm{Si}^{-}} \mathrm{H}\right), 0.33 \mathrm{eV}$ for $\left(\mathrm{Cu}_{\mathrm{Si}^{-}}-\mathrm{H}_{\mathrm{bc}}-\mathrm{Si}_{\mathrm{Si}}\right)$, and $0.73 \mathrm{eV}$ for $\left(\mathrm{Cu}_{\mathrm{Si}^{-}}-\mathrm{Si}_{\mathrm{Si}^{-}} \mathrm{H}\right)$, using 65-atom supercells. The charge state has very little effect on these energies.

There is a significant binding energy between $\mathrm{Cu}_{\mathrm{Si}}$ and interstitial $\mathrm{H}$. The reaction $\mathrm{Cu}_{\mathrm{Si}}^{0}+\mathrm{H}_{\mathrm{bc}}^{0} \rightarrow\left(\mathrm{Cu}_{\mathrm{Si}^{-}}-\mathrm{H}\right)_{C_{s}}^{0}+\mathrm{Si}_{\mathrm{Si}}$ yields $1.73 \mathrm{eV}$ for the smaller size supercell. In the larger size supercell the reaction yields $1.71 \mathrm{eV}$ when $\mathrm{Cu}_{\mathrm{Si}}$ is constrained to have $T_{d}$ symmetry, falling to $1.67 \mathrm{eV}$ when the constraint is removed. These values are somewhat smaller than the binding energy calculated by West et al.: their result is $2.46 \mathrm{eV}$ (Ref. 19).

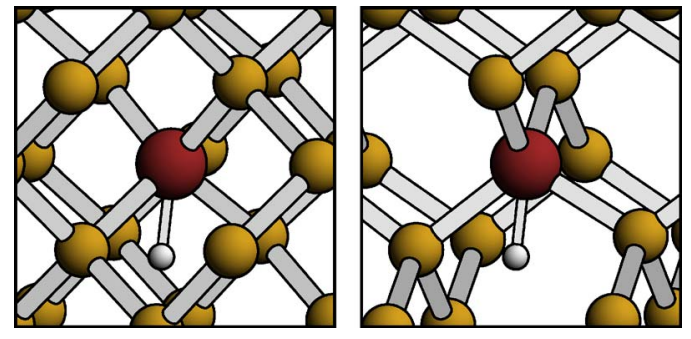

FIG. 2. (Color online) Two views of the $C_{s}$ symmetry copper monohydride complex in Si. The atom identities follow a similar pattern to Fig. 1.

Two different structures with nearly equal energies in each charge state have been found for copper dihydride $\mathrm{Cu}_{\mathrm{Si}^{-}}-\mathrm{H}_{2}$. One of them has $C_{2}$ symmetry while the other has $C_{s}$ symmetry. The rotation axis for the $C_{2}$-symmetry structure is the [100] axis that passes through the copper atom and between the two hydrogen atoms (see Fig. 4). The mirror plane for the $C_{s}$-symmetry structure is the (100) plane that contains the copper atom and has the two hydrogen atoms in equivalent positions either side of it (see Fig. 5).

The relative energies of these two defects depend on their charge state. When the defects have a charge of -2 their energies are equal to the accuracy of the method. In the charge states $-1,0$, and +1 the energy of $C_{2}$-symmetry structure is estimated to be lower than that for the $C_{s}$-symmetry structure by $0.02,0.06$, and $0.11 \mathrm{eV}$, respectively. For both sizes of supercell, the binding energy of a second hydrogen atom to a copper-monohydride complex $\left(\mathrm{Cu}_{\mathrm{Si}^{-}}-\mathrm{H}\right)_{C_{s}}^{0}+\mathrm{H}_{\mathrm{bc}}^{0}$ $\rightarrow\left(\mathrm{Cu}_{\mathrm{Si}^{\mathrm{i}}} \mathrm{H}_{2}\right)_{C_{2}}^{0}+\mathrm{Si}_{\mathrm{Si}}$ is estimated to be $1.65 \mathrm{eV}$, a value only slightly less than that for the first $\mathrm{H}$ atom. West et al. ${ }^{19}$ also report that the binding energy of the second $\mathrm{H}$ atom is about $0.1 \mathrm{eV}$ smaller than the first: their result is $2.32 \mathrm{eV}$. In annealing experiments, Knack measured the binding energy to be $1.2 \mathrm{eV}$ (Ref. 5). He notes that in $n$-type material $\mathrm{Cu}_{\mathrm{Si}}-\mathrm{H}$ and $\mathrm{Cu}_{\mathrm{Si}} \mathrm{H}_{2}$ complexes should be negatively charged and that interstitial $\mathrm{H}$ atoms will be in the +1 charge state. According to our calculations, the reaction $\left(\mathrm{Cu}_{\mathrm{Si}}-\mathrm{H}\right)_{C_{s}}^{-2}+\mathrm{H}_{\mathrm{bc}}^{+1}$ $\rightarrow\left(\mathrm{Cu}_{\mathrm{Si}^{-}}-\mathrm{H}_{2}\right)_{C_{2}}^{-1}+\mathrm{Si}_{\mathrm{Si}}$ yields 1.25 or $1.40 \mathrm{eV}$ for small and large supercells, respectively. Here, we have taken the charge states to be those that the model defects have when the Fermi level $\mu_{e}$ is at the bottom of the conduction band.

A third hydrogen atom added to the copper dihydride complexes is calculated to bind with an energy of about 1.76 $\mathrm{eV}$ to form copper trihydride complexes $\mathrm{Cu}_{\mathrm{Si}^{-}} \mathrm{H}_{3}$. The opti-
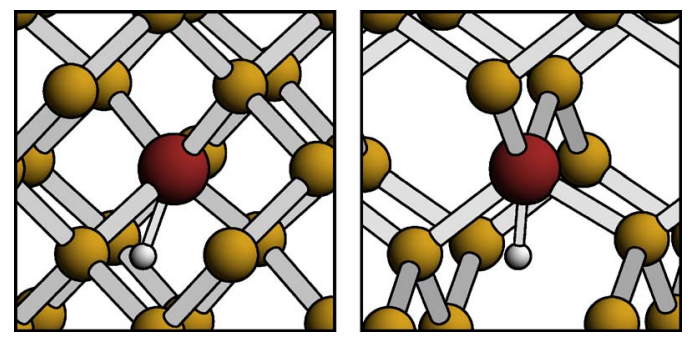

FIG. 3. (Color online) Two views of the $C_{1}$ symmetry copper monohydride complex in Si. The atom identities follow a similar pattern to Fig. 1. 


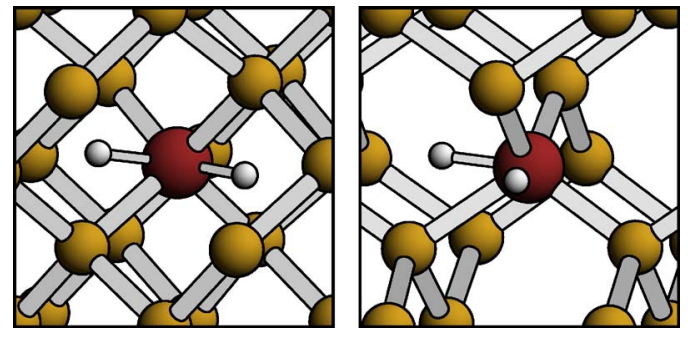

FIG. 4. (Color online) Two views of the $C_{2}$ symmetry copper dihydride complex in $\mathrm{Si}$. The atom identities follow a similar pattern to Fig. 1 with the largest size atom near the center being $\mathrm{Cu}$, surrounded by $\mathrm{Si}$ atoms, except there are now two $\mathrm{H}$ atoms attached left and right of the $\mathrm{Cu}$ in the left-hand view.

mized structure for a model defect based on the $C_{2}$-symmetry $\mathrm{Cu}_{\mathrm{Si}^{-}}-\mathrm{H}_{2}$ complex has $C_{1}$ symmetry (see Fig. $6)$. Its energy is essentially the same as for a model defect based on the $C_{s}$-symmetry structure $\mathrm{Cu}_{\mathrm{Si}}-\mathrm{H}_{2}$ complex: in the neutral state this is higher by only $0.02 \mathrm{eV}$ which is probably not significant. This defect also has very nearly $C_{S}$ symmetry, yet is not constrained to be so (see Fig. 7). One of the three hydrogen atoms lies on the mirror plane: this is a (110) plane as described previously. It is not possible to resolve whether the very small asymmetry is real or due to numerical noise.

West et al. ${ }^{19}$ estimate the binding energy of the third hydrogen atom to be $2.42 \mathrm{eV}$. They also examine the possibility of adding a fourth hydrogen atom. This, they find, is very weakly bound and they conclude that the complex is not likely to form. A full written description of the structures and symmetries of the copper-hydrogen complexes modeled by West et al. ${ }^{19}$ is not given. From visual inspection of their figures it appears that they are similar to ours.

\section{G. Electrical levels of copper-hydrogen complexes}

Knack et al. have observed a set of six electrical levels in their experiments on silicon containing copper and hydrogen impurities that they conclude are due to defect complexes containing both copper and hydrogen. ${ }^{5-7}$ Evidence for the identities of the defects responsible for these six levels comes from a careful analysis of their responses to various thermal, chemical, and electrical treatments, including estimating their depth distributions within the specimens. One of the defect complexes has three levels very similar to those of $\mathrm{Cu}_{\mathrm{Si}}$. It is identified as $\mathrm{Cu}_{\mathrm{Si}}-\mathrm{H}$. The measured energies of the three levels for this defect are $E_{d}(0 /+1)=E_{v}+0.207 \mathrm{eV}$, $E_{d}(-1 / 0)=E_{v}+0.54 \mathrm{eV}$, and $E_{d}(-2 /-1)=E_{c}-0.360 \mathrm{eV}$. A second complex identified as $\mathrm{Cu}_{\mathrm{Si}^{-}}-\mathrm{H}_{2}$ has two acceptor levels at $E_{d}(-1 / 0)=E_{v}+0.27 \mathrm{eV}$ and $E_{d}(-2 /-1)=E_{c}-0.254 \mathrm{eV}$. The identity of the defect responsible for the sixth level in the set, an acceptor near midgap at $E_{d}(-1 / 0)=E_{v}$ $+0.506 \mathrm{eV}$, appears to be different from the others, and cannot be deduced from its properties. Knack et al. also infer from their data that there are electrically neutral, electronically passive copper-hydrogen complexes present which contain three or more hydrogen atoms.

The calculated configuration energies for our model copper-hydrogen complexes are given in Table V. Given that

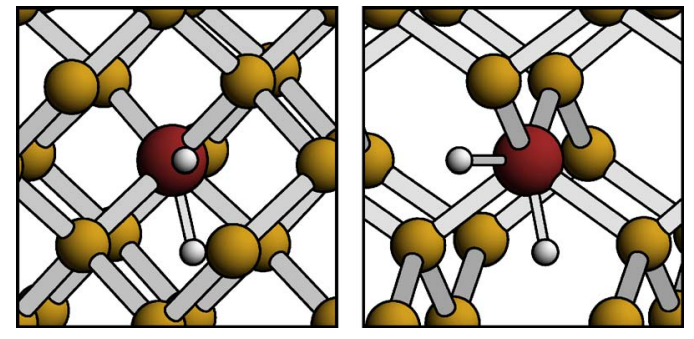

FIG. 5. (Color online) Two views of the $C_{s}$ symmetry copper dihydride complex in $\mathrm{Si}$. The atom identities are again in a similar pattern to those in the previous pictures where the $\mathrm{Cu}$ atom is at the center with two small-sized $\mathrm{H}$ atoms attached to it.

the total energies of the different forms of each of the three basic types of these defects are not significantly different from one another in each charge state, structure has little effect on $C_{d}\left(q / q^{\prime}\right)$; hence, it is not necessary to repeat the calculations for all forms of the defects in the larger size supercell.

For $d=\mathrm{Cu}_{\mathrm{Si}^{-}} \mathrm{H}$, according to the calculations, there are three levels in the bandgap with energies fairly close to the three corresponding levels for $\mathrm{Cu}_{\mathrm{Si}}$. However, compared with experiment, the relative energies are slightly different.

The calculated energy for the donor level of the complex (with $C_{s}$ symmetry) in the smaller supercell is $E_{d}(0 /+1)$ $\approx E_{v}+0.07 \mathrm{eV}$, and $E_{d}(0 /+1) \approx E_{v}+0.08 \mathrm{eV}$ in the larger supercell, with respect to pure silicon supercells. Compared with the measured donor level for $m=\mathrm{Cu}_{\mathrm{Si}}\left(C_{1}\right.$ or $D_{2 d}$ symmetry), the calculated energy differences in the smaller and larger supercells, respectively, are $D_{d}(0 /+1) \approx-0.15 \mathrm{eV}$ and $D_{d}(0 /+1) \approx-0.10 \mathrm{eV}$, while the observed levels are equal.

The calculated energy of the single acceptor level $E_{d}(-1 / 0)$ of the complex is essentially the same as it is for $\mathrm{Cu}_{\mathrm{Si}}\left(D_{d}(-1 / 0) \approx+0.01 \mathrm{eV}\right.$ in both sizes of supercells $)$. In Knack's experiments the acceptor level of the complex is about $0.06 \mathrm{eV}$ above the acceptor level of $\mathrm{Cu}_{\mathrm{Si}}$.

The double acceptor level, according to our calculations, is at $E_{d}(-2 /-1) \approx E_{c}-0.62 \mathrm{eV}$ in the smaller supercell, and $E_{d}(-2 /-1) \approx E_{c}-0.39 \mathrm{eV}$ in the larger supercell with respect to pure silicon supercells. In other words, these energies with respect to $m=\mathrm{Cu}_{\mathrm{Si}}$ are $D_{d}(-2 /-1) \approx-0.10 \mathrm{eV}$ and $D_{d}(-2 /-1) \approx-0.15 \mathrm{eV}$, respectively. This energy difference according to Knack's measurements is $D_{d}(-2 /-1)$ $=-0.087 \mathrm{eV}$. Our simulations appear to suggest that there could be a double donor level at $E_{d}(+1 /+2) \approx E_{v}$ $+0.02 \mathrm{eV}(m=\mathrm{Si})$. It is likely that this is only an artifact of the theoretical approximations, which become poorer with increasing charge state.

The results for $\mathrm{Cu}_{\mathrm{Si}}$ and $\mathrm{Cu}_{\mathrm{Si}}-\mathrm{H}$ taken together provide a crude indication of the accuracy of the method. The standard deviation (root mean square error) with respect to the measured energies for all six levels of these two defects, calculated in the large-size supercells, using ideal silicon supercells as the reference state is $0.11 \mathrm{eV}$. If $\mathrm{Cu}_{\mathrm{Si}}$ is used as the reference potential or marker to calculate the energies of the three levels of $\mathrm{Cu}_{\mathrm{Si}^{-}} \mathrm{H}$, then the standard deviation is $0.07 \mathrm{eV}$. Clearly, this is by no means a rigorous analysis; however, it does give some impression of the magnitude of the errors 

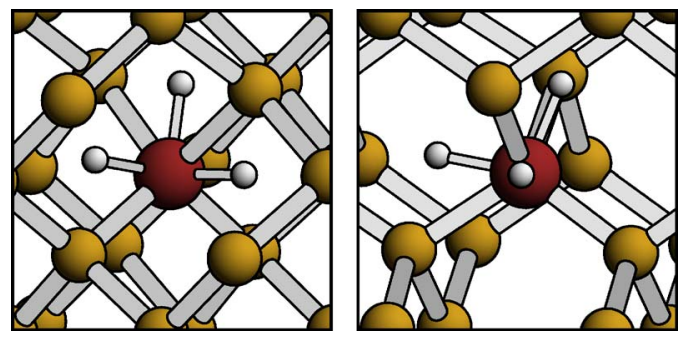

FIG. 6. (Color online) Two views of the copper trihydride complex in $\mathrm{Si}$ with $C_{1}$ symmetry. As in the previous pictures, the large atom at the center is $\mathrm{Cu}$, now with three $\mathrm{H}$ attoms attached to it.

and the level of improvement that might be expected from using an empirical marker, and is in accord with our past experience of similar calculations.

Up to this point the energy levels of the model defects bear more than a passing resemblance to the ones observed experimentally. This is not the case for $d=\mathrm{Cu}_{\mathrm{Si}_{\mathrm{i}}}-\mathrm{H}_{2}$. Our calculations predict that this complex has a donor level in the lower part of the bandgap and an acceptor level slightly below midgap. The model $\mathrm{Cu}_{\mathrm{Si}}-\mathrm{H}_{2}$ complexes do not induce any other levels in the bandgap.

The calculated energies for the donor level of the $C_{2}$-symmetry complex in small and large supercells, respectively, are, $E_{d}(0 /+1) \approx E_{v}+0.18 \mathrm{eV}$ and $E_{d}(0 /+1) \approx E_{v}$ $+0.10 \mathrm{eV}$, with respect to pure silicon supercells. If we take the measured energy $E_{m}(0 /+1)=E_{v}+0.207 \mathrm{eV}$ for $m=\mathrm{Cu}_{\mathrm{Si}}$ as a marker, together with its calculated configuration energies $C_{m}(0 /+1)$ (with $C_{1}$ or $D_{2 d}$ symmetry), then the calculated energies with respect to this are $E_{d}(0 /+1) \approx E_{v}$ $+0.16 \mathrm{eV}$ and $E_{d}(0 /+1) \approx E_{v}+0.15 \mathrm{eV}$ in the smaller and larger supercells.

The calculated acceptor level is at $E_{d}(-1 / 0) \approx E_{c}$ $-0.78 \mathrm{eV}$ (small supercell) and $E_{d}(-1 / 0) \approx E_{c}-0.57 \mathrm{eV}$ (large supercell) with respect to pure silicon; while using $m$ $=\mathrm{Cu}_{\mathrm{Si}}$ and $E_{g}=1.17 \mathrm{eV}$, we estimate $E_{d}(-1 / 0) \approx E_{c}$ $-0.78 \mathrm{eV}$ (small supercell) and $E_{d}(-1 / 0) \approx E_{c}-0.76 \mathrm{eV}$ (large supercell). As this energy is slightly larger than $E_{g}$ for the larger supercell, and theory does not appear to match experiment, there is uncertainty where the true level is, so we must consider carefully whether or not to make $E_{v}$ the reference when comparing with pure silicon supercells. If this is done, then based on our previous experience with $\mathrm{Cu}_{\mathrm{Si}}$ and

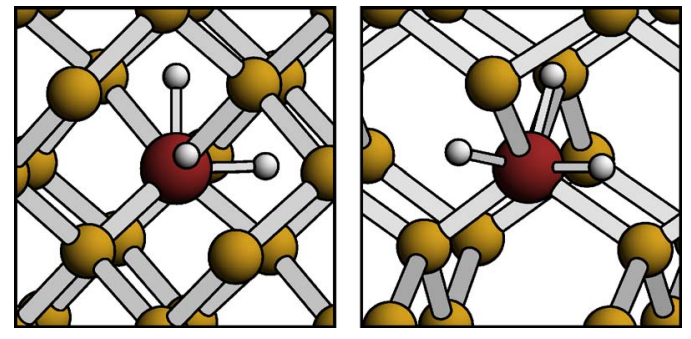

FIG. 7. (Color online) Two views of the copper trihydride complex in $\mathrm{Si}$ with approximately $C_{s}$ symmetry. The atom identities are as described previously.

$\mathrm{Cu}_{\mathrm{Si}} \mathrm{H}$, the acceptor level looks too close to the donor. In this case, therefore, the true level lies a little higher than this, but below midgap. Thus, changing the reference from $E_{v}$ to $E_{c}$ does not change our conclusion significantly.

Both forms of the $\mathrm{Cu}_{\mathrm{Si}}-\mathrm{H}_{3}$ appear to be electrically inactive defects. The energy needed to remove one electron from the complex with $C_{s}$ symmetry is nearly the same as for a 64-atom supercell of pure silicon. The complex with nearly $C_{1}$ symmetry with a charge +1 has a total energy $\approx 0.06 \mathrm{eV}$ lower than the $C_{s}$ symmetry configuration. This gives it a donor level $E_{d}(0 /+1) \approx E_{v}+0.04 \mathrm{eV}$ with respect to pure $\mathrm{Si}$. This is too close to the top of the valence band to resolve definitely whether or not it is real or an artifact of the method. The theoretical acceptor levels of both types of the defect lie above the bottom of the conduction band.

\section{SUMMARY AND CONCLUSIONS}

Using the AIMPRO method, we have explored the structural, energetic, and electronic properties of several defects in silicon containing copper and hydrogen impurities. A wellknown weakness of the method is the underestimation of semiconductor bandgaps. This is demonstrated for silicon by our results. Electrical levels are also difficult to calculate accurately. A strategy based on differences in total energies of supercells is developed that aims to give meaningful results by careful consideration of each particular situation.

The known properties of isolated intersitial hydrogen $\mathrm{H}_{i}$ are reproduced well compared with both previous theory and experiment. This includes its structure, electrical levels, and negative- $U$ behavior.

TABLE V. Configuration energies $C_{d}\left(q / q^{\prime}\right)$ in electron volts for copper-hydrogen complexes in $\mathrm{Si}$. The same remarks about numerical versus real precision as in Table I and Sec. II apply here.

\begin{tabular}{|c|c|c|c|c|c|c|c|}
\hline \multirow[b]{2}{*}{$d \backslash q / q^{\prime}$} & \multicolumn{4}{|c|}{$2 \times 2 \times 2$ supercell } & \multicolumn{3}{|c|}{$3 \times 3 \times 3$ supercell } \\
\hline & $+1 /+2$ & $0 /+1$ & $-1 / 0$ & $-2 /-1$ & $0 /+1$ & $-1 / 0$ & $-2 /-1$ \\
\hline$\left(\mathrm{Cu}_{\mathrm{Si}^{-}}-\mathrm{H}\right)_{C_{2 v}}$ & 5.052 & 5.097 & 5.331 & 5.405 & & & \\
\hline$\left(\mathrm{Cu}_{\mathrm{Si}}-\mathrm{H}\right)_{C_{s}}$ & 5.059 & 5.109 & 5.342 & 5.407 & 5.252 & 5.392 & 5.492 \\
\hline$\left(\mathrm{Cu}_{\mathrm{Si}^{-}}-\mathrm{H}\right)_{C_{1}}$ & 5.060 & 5.109 & 5.344 & 5.406 & & & \\
\hline$\left(\mathrm{Cu}_{\mathrm{Si}}-\mathrm{H}_{2}\right)_{C_{2}}$ & 4.915 & 5.216 & 5.243 & 6.100 & 5.279 & 5.312 & 5.991 \\
\hline$\left(\mathrm{Cu}_{\mathrm{Si}}-\mathrm{H}_{2}\right)_{C_{s}}$ & 4.920 & 5.167 & 5.202 & 6.089 & & & \\
\hline$\left(\mathrm{Cu}_{\mathrm{Si}}-\mathrm{H}_{3}\right)_{C_{s}}$ & 5.029 & 5.043 & 6.102 & 6.091 & & & \\
\hline$\left(\mathrm{Cu}_{\mathrm{Si}^{-}}-\mathrm{H}_{3}\right)_{C_{1}}$ & 5.077 & 5.081 & 6.109 & 6.102 & & & \\
\hline
\end{tabular}


Interstitial copper $\mathrm{Cu}_{i}$, also in agreement with experiments, according to the calculations is a shallow donor. The only known properties for isolated, substitutional copper atoms $\mathrm{Cu}_{\mathrm{Si}}$ are its electrical levels. The theoretical method again in agreement with experiment reproduces the measured energies of the defect-induced electrical levels. In addition to this it is predicted, as anticipated, that the defect is susceptible to the Jahn-Teller effect.

While the electrical levels for $\mathrm{Cu}_{\mathrm{Si}}$ have been well established by a number of successively improved measurements, far fewer data for copper-hydrogen complexes exist. In broad terms, the model copper-hydrogen complexes with lowest energy have up to three hydrogen atoms attached close to the copper atom in the open interstitial spaces nearest to the cubic directions of the silicon crystal. There are several nearly equal energy minima for the hydrogen atoms resulting in a number of different structures. $\mathrm{Cu}_{\mathrm{Si}}-\mathrm{H}$ has three distinct variants while $\mathrm{Cu}_{\mathrm{Si}^{-}} \mathrm{H}_{2}$ and $\mathrm{Cu}_{\mathrm{Si}^{-}}-\mathrm{H}_{3}$ have two each. It is reasonable to expect that the activation energies for transformation of the complexes from one form to another are fairly low. In all cases the binding energy per hydrogen atom when the species are neutral is similar at about $1.7 \mathrm{eV}$. This figure is a little lower for the formation of $\mathrm{Cu}_{\mathrm{Si}^{-}}-\mathrm{H}_{2}$ from $\mathrm{Cu}_{\mathrm{Si}}-\mathrm{H}$ and $\mathrm{H}$ in $n$-type material where the charge states of the species need to be taken into account. When this is done, the theoretical estimate for the binding energy is only $\sim 0.1 \mathrm{eV}$ higher than the measured amount, $1.2 \mathrm{eV}$.

Theory and experiment remain in good agreement on the character and energies of the electrical levels of $\mathrm{Cu}_{\mathrm{Si}}-\mathrm{H}$ and $\mathrm{Cu}_{\mathrm{Si}^{-}}-\mathrm{H}_{3}$. The monohydride complex has three levels similar to $\mathrm{Cu}_{\mathrm{Si}}$ while the trihydride appears to be a passive defect. However, for $\mathrm{Cu}_{\mathrm{Si}_{\mathrm{i}}}-\mathrm{H}_{2}$ the electronic levels of the model complex differ from the experimental assignments. Theory predicts the complex has a deep donor level in the lower part of the bandgap, between the predicted donor levels for $\mathrm{Cu}_{\mathrm{Si}}$, and $\mathrm{Cu}_{\mathrm{Si}^{-}} \mathrm{H}$. We suggest this near degeneracy may explain why no donor level has been observed for $\mathrm{Cu}_{\mathrm{Si}^{-}}-\mathrm{H}_{2}$. The model complex also has an acceptor level above the donor and below midgap. No evidence is found for the existence of a double acceptor level for $\mathrm{Cu}_{\mathrm{Si}^{-}}-\mathrm{H}_{2}$, or any other level within
TABLE VI. Summary of the "best" estimates for the energies of electrical levels in electron volts for model defects of copper and hydrogen impurities in silicon calculated with respect to pure silicon supercells.

\begin{tabular}{lccc}
\hline \hline$d \backslash q / q^{\prime}$ & $0 /+1$ & $-1 / 0$ & $-2 /-1$ \\
\hline $\mathrm{H}_{i}$ & $E_{c}-0.07$ & $E_{c}-0.50$ & \\
$\mathrm{Cu}_{i}$ & $E_{c}-0.03$ & & \\
$\mathrm{Cu}_{\mathrm{Si}}$ & $E_{v}+0.17$ & $E_{c}-0.50$ & $E_{c}-0.24$ \\
$\mathrm{Cu}_{\mathrm{Si}}-\mathrm{H}$ & $E_{v}+0.08$ & $E_{c}-0.49$ & $E_{c}-0.39$ \\
$\mathrm{Cu}_{\mathrm{Si}}-\mathrm{H}_{2}$ & $E_{v}+0.10$ & $E_{c}-0.57$ & \\
\hline \hline
\end{tabular}

the bandgap. The calculated total energy for this complex in the -2 charge state places its energy level well above the conduction band minimum. We can find no explanation among our models for the level observed by Knack et al. at $E_{c}-0.254 \mathrm{eV}$. The acceptor near midgap at $E_{v}+0.506 \mathrm{eV}$ also remains unexplained. Further calculations for other model defects will be needed to resolve these issues.

Finally, Table VI provides a summary of what may be considered our best estimates for the energies of electrical levels for the model defects calculated according to the strategy adopted for this work.

\section{ACKNOWLEDGMENTS}

This research has been supported by Lappeenranta University of Technology, and the Academy of Finland through its Centers of Excellence Programme 2000-2005. Computer resources have been provided by the National Supercomputer Centre in Sweden (NSC), the Centre for Scientific Computing (CSC) in Finland, and Computer Services for Academic Research (CSAR) in the United Kingdom. S.Ö. and C.D.L. also thank the Swedish Research Council (VR) for providing financial support. Finally, C.D.L. and R.M.N. wish to express their gratitude to Maria Ganchenkova at HUT for helpful discussions.
*Email address: cdl@fyslab.hut.fi

${ }^{1}$ A. A. Istratov, C. Flink, H. Hieslmair, E. R. Weber, and T. Heiser, Phys. Rev. Lett. 81, 1243 (1998).

${ }^{2}$ K. Lemke, Phys. Status Solidi A 95, 665 (1986).

${ }^{3}$ S. D. Brotherton, J. R. Ayres, A. Gill, H. W. van Kesteren, and F. J. A. M. Greidanus, J. Appl. Phys. 62, 1826 (1987).

${ }^{4}$ A. A. Istratov, H. Hieslmair, C. Flink, T. Heiser, and E. R. Weber, Appl. Phys. Lett. 71, 2349 (1997).

${ }^{5}$ S. Knack, Ph.D. thesis, der Fakultät Mathematik und Naturwissenschaften, der Technischen Universität Dresden, Germany (2002), Logos Verlag, Berlin.

${ }^{6}$ S. Knack, J. Weber, and H. Lemke, Physica B 273-274, 387 (1999).

${ }^{7}$ S. Knack, J. Weber, H. Lemke, and H. Riemann, Phys. Rev. B 65, 165203 (2002).
${ }^{8}$ S. Weiss, R. Beckmann, and R. Kassing, Appl. Phys. A 50, 151 (1990).

${ }^{9}$ N. Yarykin, J.-U. Sachse, H. Lemke, and J. Weber, Phys. Rev. B 59, 5551 (1999).

${ }^{10}$ E. Ö. Sveinbjörnsson and O. Engström, Phys. Rev. B 52, 4884 (1995).

${ }^{11}$ L. Rubaldo, P. Deixler, I. D. Hawkins, J. Terry, D. K. Maude, J. C. Portal, J. H. Evans-Freeman, L. Dobaczewski, and A. R. Peaker, Mater. Sci. Eng., B 58, 126 (1999).

${ }^{12}$ J.-U. Sachse, E. Ö. Sveinbjörnsson, W. Jost, J. Weber, and H. Lemke, Phys. Rev. B 55, 16176 (1997).

${ }^{13}$ J.-U. Sachse, W. Jost, J. Weber, and H. Lemke, Appl. Phys. Lett. 71, 1379 (1997).

${ }^{14}$ G. D. Watkins, Physica B \& C 117-118, 9 (1983).

${ }^{15}$ S. K. Estreicher and J. L. Hastings, Mater. Sci. Eng., B 58, 155 
(1999).

${ }^{16}$ S. K. Estreicher, Physica B 273-274, 424 (1999).

${ }^{17}$ S. K. Estreicher, Phys. Rev. B 60, 5375 (1999).

${ }^{18}$ S. K. Estreicher, D. West, and P. Ordejón, Solid State Phenom. 82-84, 341 (2002).

${ }^{19}$ D. West, S. K. Estreicher, S. Knack, and J. Weber, Phys. Rev. B 68, 035210 (2003).

${ }^{20} \mathrm{R}$. Jones and P. R. Briddon, The ab Initio Cluster Method and the Dynamics of Defects in Semiconductors, Semiconductors and Semimetals Vol. 51A (Academic Press, Boston, 1998), Chap. 6.

${ }^{21}$ P. R. Briddon and R. Jones, Phys. Status Solidi B 217, 131 (2000).

${ }^{22}$ J. Coutinho, R. Jones, P. R. Briddon, and S. Öberg, Phys. Rev. B 62, 10824 (2000).

${ }^{23}$ H. J. Monkhorst and J. D. Pack, Phys. Rev. B 13, 5188 (1976).

${ }^{24}$ C. Hartwigsen, S. Goedecker, and J. Hutter, Phys. Rev. B 58, 3641 (1998).

${ }^{25}$ J. P. Perdew and Y. Wang, Phys. Rev. B 45, 13244 (1992).

${ }^{26}$ S. B. Zhang and J. E. Northrup, Phys. Rev. Lett. 67, 2339 (1991).

${ }^{27}$ J. E. Northrup and S. B. Zhang, Phys. Rev. B 47, R6791 (1993).
${ }^{28}$ S. Pöykkö, M. J. Puska, and R. M. Nieminen, Phys. Rev. B 53, 3813 (1996).

${ }^{29}$ T. Mattila and R. M. Nieminen, Phys. Rev. B 54, 16676 (1996).

${ }^{30}$ C. G. Van de Walle, S. Limpijumnong, and J. Neugebauer, Phys. Rev. B 63, 245205 (2001).

${ }^{31}$ G. Makov and M. C. Payne, Phys. Rev. B 51, 4014 (1995).

${ }^{32}$ M. Methfessel and A. T. Paxton, Phys. Rev. B 40, 3616 (1989).

${ }^{33}$ S. K. Estreicher, Mater. Sci. Eng., R. 14, 319 (1995).

${ }^{34}$ C. G. Van de Walle, P. J. H. Denteneer, Y. Bar-Yam, and S. T. Pantelides, Phys. Rev. B 39, 10791 (1989).

${ }^{35}$ K. Bonde Nielsen, B. Bech Nielsen, J. Hansen, E. Andersen, and J. U. Andersen, Phys. Rev. B 60, 1716 (1999).

${ }^{36}$ C. Herring, N. M. Johnson, and C. G. Van de Walle, Phys. Rev. B 64, 125209 (2001).

${ }^{37}$ J. S. Lord, S. F. J. Cox, M. Charlton, D. P. Van der Werf, R. L. Lichti, and A. Amato, J. Phys.: Condens. Matter 40, S4739 (2004).

${ }^{38}$ M. J. Puska, S. Pöykkö, M. Pesola, and R. M. Nieminen, Phys. Rev. B 58, 1318 (1998). 